\title{
As abordagens terapêuticas psicológicas na qualidade de vida dos autistas: Revisão
}

\section{de literatura}

\author{
Psychological therapeutic approaches to the quality of life of autistic people: Literature review \\ Enfoques psicológicos terapéuticos para la calidad de vida de las personas autistas: Revisión de la
} literatura

Recebido: 06/06/2021 | Revisado: 13/06/2021 | Aceito: 23/06/2021 | Publicado: 10/07/2021

\author{
Antonia de Jesus da Silva Faustino \\ ORCID: https://orcid.org/0000-0001-8363-951X \\ Faculdade de Ensino Superior do Piauí, Brasil \\ E-mail: ajsfimoveis@gmail.com \\ Salma Suellen Ingelsrud Leal \\ ORCID: https://orcid.org/0000-0002-6086-6151 \\ Faculdade de Ensino Superior do Piauí, Brasil \\ E-mail: salmaleal@grupomagister.com \\ Érica Vanessa Rodrigues da Silva \\ ORCID: https://orcid.org/0000-0003-1283-374X \\ Faculdade de Ensino Superior do Piauí, Brasil \\ E-mail:ericavanessa@grupomagister.com \\ Ruth Raquel Soares de Farias \\ ORCID: https://orcid.org/0000-0002-0988-0900 \\ Universidade Federal do Piauí, Brasil \\ E-mail: ruthraquelsf@gmail.com
}

\begin{abstract}
Resumo
Este artigo estudo sobre as abordagens terapêuticas psicológicas na qualidade de vida dos autistas. Tem como objetivo geral verificar as terapias utilizadas pelo psicólogo, que contribuem para uma melhor qualidade destas pessoas. A pesquisa pontua, em seus objetivos específicos, entre outros aspectos, apresentar modelos e/ou estratégias de intervenções utilizadas por psicólogos junto ao autismo; identificar as características das intervenções realizado pelas abordagens: Terapia Cognitivo Comportamental (TCC); Análise do Comportamento Aplicada (ABA); Abordagens Humanistas e Fenomenológicas e a Psicanalise além de analisar a eficácia das abordagens psicológicas no acompanhamento clínico e na qualidade de vida dos autistas. As abordagens terapêuticas visam reduzir comprometimento do autista, auxiliando os indivíduos a conquistarem a sua independência. Foi realizada uma revisão de literatura sistemática, com utilização de livros, teses, além de artigos nos anos de (2015-2020) dos bancos dos dados da Scientific Electronic Library Online - Scielo, Literatura Latino-Americana e do Caribe em Ciências da Saúde (LILACS) e MED LINE (Medical LiteratureAnalysisandRetrieval System OnLine) em língua Portuguesa, Inglesa e Espanhola em busca de publicações relevantes que discutissem o assunto a ser de fundamental interesse que é, autismo, psicologia, abordagens terapêuticas e qualidade de vida. Diante do exposto, conclui-se que existe um amplo campo de técnicas e abordagens, ressalta-se que, no caso do autismo, exige do profissional uma diretividade mais ampla com o objetivo de oferecer propostas de intervenções eficazes e que incentivem, na medida do possível, sua independência.
\end{abstract}

Palavras-chaves: Psicologia; Terapias; Bem-estar.

\begin{abstract}
The present work discusses the psychological therapeutic approaches in the quality of life of the autistic, its general objective is to verify the therapeutic approaches used by the psychologist, which contribute to a better quality of life for the autistic. The research points,in its specific objectives, models and / or strategies to present the interventions used by psychologists with autism; identify the characteristics of the interventions carried out by the approaches, Cognitive Behavioral Therapy (CBT), Applied Behavior Analysis (ABA), Humanistic and Phenomenological Approaches and Psychoanalysis and analyze the effectiveness of psychological approaches in clinical follow-up and in the quality of life of autistic people. Interventions and therapeutic approaches aim toreduce impairment, helping individuals to overcome this difficulty. A narrative literature review (RNL) was carried out, using books, theses, as well as articles in the years (2015-2020) from the databases of the Scientific Electronic Library Online - Scielo, Latin American and Caribbean Literature in Sciences of Health (LILACS) and MED LINE (Medical Literature Analysis and Retrieval System On Line) in Portuguese, English and Spanish in search of relevant publications that discuss the
\end{abstract}


subject of fundamental interest, which is, autism, psychology, therapeutic approaches and quality of life. life. In view of the above, it is concluded that there is a wide field of technical approaches, it is emphasized that, in the case of autism, greater directivity is required from the psychotherapist, with the aim of offering intervention proposals that encourage, as far as possible, the child's compromised contact process.

Keywords: Psychology; Therapies; Welfare.

\section{Resumen}

Este artículo analizalos enfoques terapéuticos psicológicos para lacalidad de vida de las personas autistas. Su objetivo general es verificar las terapias utilizadas por el psicólogo, que contribuyan a una mejorcalidad de estas personas. La investigaciónapunta, en sus objetivos específicos, entre otros aspectos, presentar modelos y / o estrategias de intervención utilizadas por psicólogos con autismo; identificar las características de las intervenciones realizadas por los enfoques: Terapia cognitivo-conductual (TCC); Análisis de comportamiento aplicado (ABA); Enfoques humanistas, fenomenológicos y psicoanálisis, además de analizarlaefectividad de los enfoques psicológicos enelseguimiento clínico y lacalidad de vida de los autistas. Los enfoques terapéuticostienen como objetivo reducirel deterioro autista, ayudando a las personas a ganarsuindependencia. Se realizó una revisión bibliográfica sistemática, utilizando libros, tesis y artículos de losaños (2015-2020) de la Biblioteca Electrónica Científica Online - Scielo, bases de datos de Ciencias de laSalud de América Latina y el Caribe (LILACS) y MED LINE (Análisis y Retrieval System OnLine) enportugués, inglés y españolen busca de publicaciones relevantes que abordenel tema de interés fundamental, que es el autismo, lapsicología, los enfoques terapéuticos y lacalidad de vida. Dado lo anterior, se concluye que existe un amplio campo de técnicas y enfoques, es de destacar que, enel caso del autismo, se requieredelprofesional una directiva más amplia para poder ofrecerpropuestas de intervenciones efectivas y que incentiven, como loposible, suindependencia.

Palabras clave: Psicología; Terapias; Bienestar.

\section{Introdução}

O transtorno do espectro do autismo (TEA), também conhecido como autismo, é um transtorno neurológico que pode ser desenvolvido nos primeiros três anos de vida e é caracterizado por afetar a interação social, linguagem e padrões restritos e repetitivos de comportamento.de coordenação motora. Para Paiva Júnior (2021), é uma condição caracterizada por déficits persistentes de comunicação (comunicação social e verbal e não verbal) e no comportamento (interesse restrito e ações repetitivas) (Teixeira, 2016).

Com relação as alterações da cognição e comportamento nos autistas revelam que em grande maioria estão associadas ao atraso no seu desenvolvimento, sendo importante o uso de intervenções que proporcionem melhorias nas funções biológicas e comportamentais que envolvem essas habilidades, sejam elas simples ou complexas. (Alvarenga, 2017).

Considerando estas alterações confirmamos a necessidade de se utilizar uma abordagem terapêutica que pode ser realizada pelo psicólogo por meio de algumas abordagens como a Terapia Cognitivo Comportamental (TCC), Análise do Comportamento Aplicada (ABA), as Abordagens Humanistas e Fenomenológicas e a Psicanalise (Chaim, 2020).

No viés psicanalítico existem muitas indagações sobre o que vem a ser o autismo, não se caracterizando como estrutura clínica neurótica ou psicótico. Dentro da Psicanálise, a intervenção de pessoas com autismo vem apresentando resultados, significativos embora sua origem ainda não tenha causa específica ou definida (Gonçalves et al., 2017).

Em relação à abordagem fenomenológica humanística, apontou que é ideal observar como uma pessoa entende a experiência, porque a compreensão de cada um de sua própria experiência é o mais importante, no autismo e outro tipo de condição humana. É importante que "a singularidade dessa pessoa seja reconhecida, independentemente da condição patológica". A fenomenologia é pautada na abordagem centrada no ser humano visa enfatizar a experiência, buscar compreender o fenômeno e verificar a percepção do psicoterapeuta e o outro, diante da essência da psicoterapia, ou seja, o encontro de duas pessoas (Chaim, 2020).

Sobre a Fenomenologia e o Humanismo revela que trabalham com situações atuais, ou seja, com aqui e agora. O psicólogo trata as soluções dos problemas baseados no presente diferenciando-se da psicanálise, que foca a partir da infância além disso, a fenomenologia também afirma que todo ser humano tem algo bom, abordam as questões positivas das pessoas, 
sendo que todas as escolhas feitas são responsabilidades de cada um. As atitudes em tudo que o ser humano venha fazer, ou ter feito, é responsabilidade dele, ele tem que ter essa consciência (Bezerra, 2018).

A Análise Aplicada do Comportamento (ABA) é um campo do conhecimento baseado no desenvolvimento, pesquisa e aplicação dos princípios básicos da ciência da análise comportamental". Existem afirmações que o ABA consiste em uma abordagem que se caracteriza como individualizada e altamente estruturada, por isso sua aplicação em indivíduos com TEA resulta em êxito (Instituto neurosaber, 2017).

No que tange a Terapia Cognitivo Comportamental (TCC), nas considerações de é uma subárea da psicologia que trata das relações entre cognição e comportamento, possuindo um aparato técnico - teórico direto, breve e específico, direcionados a situação presente, com as demandas atuais (Assunção; Silva, 2019). Com relação ao autismo Conslini, Lopes e Lopes (2019, p.38) "afirmam que a TCC apresenta resultados efetivos para o TEA, porém, adaptações são indissociáveis e há a necessidade de maior compreensão da manifestação de ansiedade nessa população.”

Este objetiva problematizar as abordagens terapêuticas psicológicas que contribuem como fator de influência na qualidade de vida do indivíduo com o TEA. É importante salientar que o autismo é um assunto que quando vem à tona, gera uma série de desafios, em diversos âmbitos, como educacional, social e no âmbito da Psicologia.

\section{Metodologia}

Neste trabalho foi desenvolvida uma revisão de literatura, com utilização de livros, teses, além de artigos dos bancos dos dados da ScientificElectronic Library Online - Scielo, Literatura Latino-Americana e do Caribe em Ciências da Saúde (LILACS) e MED LINE (Medical LiteratureAnalysisandRetrieval System OnLine) em língua Portuguesa, Inglesa e Espanhola em busca de publicações relevantes que discutissem o assunto a ser de fundamental interesse que é, autismo, psicologia, abordagens terapêuticas e qualidade de vida.

A pesquisa possui caráter amplo e objetivo de descrever o assunto proposto, de acordo com ponto de vista teórico ou contextual, analisando e interpretando produções cientificas existentes. Como propósito geral a pesquisa é de caráter exploratória, "seu planejamento tende a ser bastante flexível, pois interessa considerar os mais variados aspectos relativos ao fato ou fenômeno estudado" (Gil, 2017, p.33). Quando a natureza dos dados é uma básica de abordagem qualitativa.

Quanto aos critérios de inclusão foram catalogados os artigos que agregam aos seus objetivos, a descrição de proposta apresenta, estratégias de intervenção terapêutica visando a qualidade de vida de pessoas com TEA entre os anos de fevereiro de 2015 a abril de 2021. Exclusão foram definidos os seguintes critérios: artigos duplicados, artigos de revisão e incompletos, teses e dissertações, livros, resenhas, estudos em escolas de outros países, em locais fora do âmbito escolar, bem como estudos que não traziam informações claras sobre a metodologia.

Depois de selecionados os artigos, foi realizada a análise, com intuito de responder as seguintes informações: quais os benefícios e eficácia nas abordagens terapêuticas descritas, quais os enfoques de tratamento e além da importância das abordagens utilizadas. Uma observação sobre o processo de seleção de artigos foi a dificuldade de encontrar artigos que discursem sobre as abordagens escolhidas para construção do projeto. Assim foi um recorte alinhado descritores em pares, utilizando os operadores booleanos "AND" E "OR".

Foram encontradas 243 publicações sobre os descritores utilizados quando cruzados e considerando o objetivo da pesquisa foram selecionados 10 que atenderam os critérios de inclusão e exclusão, aplicou-se o filtro de recorte temporal nos últimos 5 anos, buscando trabalhos mais relevantes atualmente. 


\section{Resultados e Discussão}

Assim, após a leitura dos artigos, e com base nas categorias temáticas as informações foram retiradas e fichadas para a elaboração do quadro. Abaixo a tabela com os estudos que tratam do Transtorno do Espectro Autista, abordagem terapêutica e qualidade de vida, visando apresentar eficácia, importância das abordagens utilizadas

Posteriormente, analisados os artigos e teses encontradas foi construído um quadro com 10 estudos selecionados com pontuações relevantes sobre o assunto. Conforme observado no quadro 1, as estratégias de intervenção terapêutica visando a qualidade de vida de pessoas com Transtorno do Espectro Autista.

Quadro 1 - Artigos selecionados e divididos por categorias apresentado considerações acerca das estratégias de intervenção terapêutica visando a qualidade de vida de pessoas com Transtorno do Espectro Autista.

\begin{tabular}{|c|c|c|c|}
\hline AUTOR/ ANO & OBJETIVO & RESULTADOS & CONCLUSÃO \\
\hline $\begin{array}{l}\text { Steensel e Bögels } \\
(2015)\end{array}$ & $\begin{array}{l}\text { Examinar a eficácia da terapia } \\
\text { cognitivo-comportamental } \\
\text { para transtornos de ansiedade em } \\
\text { crianças com transtornos do espectro } \\
\text { do autismo (TEA) foi examinada e } \\
\text { comparada com crianças sem TEA. }\end{array}$ & $\begin{array}{l}\text { A TCC foi mais eficaz do que a lista de } \\
\text { espera para o tratamento de transtornos de } \\
\text { ansiedade e sintomas de ansiedade em } \\
\text { crianças com ASD. A diminuição da } \\
\text { gravidade dos transtornos de ansiedade } \\
\text { após a TCC não foi diferente para } \\
\text { crianças com e sem TEA. }\end{array}$ & $\begin{array}{l}\text { A TCC para transtornos de ansiedade } \\
\text { é eficaz para crianças com TEA, } \\
\text { também em longo prazo. Os ganhos } \\
\text { do tratamento podem ser um pouco } \\
\text { menores em comparação com } \\
\text { crianças sem TEA. }\end{array}$ \\
\hline $\begin{array}{l}\text { Barreto, } \\
\text { Nascimento e } \\
\text { Andrade (2015) }\end{array}$ & $\begin{array}{l}\text { Investigar a significação dos objetos } \\
\text { autísticos e transicionais no processo } \\
\text { de subjetivação destas crianças } \\
\text { submetidas ao acompanhamento } \\
\text { psicológico e os efeitos de seus } \\
\text { desdobramentos na relação } \\
\text { transferencial com o terapeuta, visto } \\
\text { que estes objetos fornecem indícios } \\
\text { sobre a elaboração (ou não) do } \\
\text { processo de separação-individuação } \\
\text { da criança frente à mãe }\end{array}$ & $\begin{array}{l}\text { A prática clínica é soberana, podendo } \\
\text { reafirmar e/ou questionar as teorias } \\
\text { existentes. }\end{array}$ & $\begin{array}{l}\text { É possível apontar avanços no quadro } \\
\text { clínico destas crianças em decorrência } \\
\text { do processo terapêutico, que podem } \\
\text { se revelar em uma maior abertura ao } \\
\text { Outro e no uso simbólico e funcional } \\
\text { destes objetos }\end{array}$ \\
\hline $\begin{array}{l}\text { Locatelli e Santos } \\
\text { (2016) }\end{array}$ & $\begin{array}{l}\text { Aborda o autismo, dando ênfase aos } \\
\text { métodos de intervenção com as } \\
\text { crianças autistas }\end{array}$ & $\begin{array}{l}\text { Resultados obtidos com tratamento da } \\
\text { equipe multidisciplinar iniciado logo após } \\
\text { o diagnóstico, contribuiu para uma } \\
\text { melhora comportamental significativa nos } \\
\text { aspectos relacionados ao convívio e a } \\
\text { interação com as pessoas ao seu redor. }\end{array}$ & $\begin{array}{l}\text { O profissional em Psicologia deve } \\
\text { conhecer as técnicas de intervenção } \\
\text { para indivíduos com TEA, entanto o } \\
\text { suporte à família e à preservação da } \\
\text { subjetividade da pessoa é de } \\
\text { primordial importância para qualquer } \\
\text { prognóstico positivo. }\end{array}$ \\
\hline Alvarenga (2017) & $\begin{array}{l}\text { Verificar as principais ações } \\
\text { terapêuticas para essa Autismo }\end{array}$ & $\begin{array}{l}\text { Os resultados mostraram que a abordagem } \\
\text { cognitivo-comportamental tem se } \\
\text { mostrado bastante eficaz em casos de } \\
\text { TEA pois trabalha aspectos da cognição e } \\
\text { mudanças comportamentais, o que } \\
\text { possibilita resultados mais satisfatórios. }\end{array}$ & $\begin{array}{l}\text { O treino de habilidades sociais pode } \\
\text { proporcionar melhora significativa no } \\
\text { quadro de autismo leve e melhorar o } \\
\text { funcionamento social do indivíduo o } \\
\text { que previne comorbidades como } \\
\text { transtornos ansiosos e depressivos que } \\
\text { ocorrem pela frustração da }\end{array}$ \\
\hline
\end{tabular}


Research, Society and Development, v. 10, n. 8, e16010816870, 2021

(CC BY 4.0) | ISSN 2525-3409 | DOI: http://dx.doi.org/10.33448/rsd-v10i8.16870

\begin{tabular}{|c|c|c|c|}
\hline & & & inadequação social. \\
\hline $\begin{array}{l}\text { Tovar e Rodriguez } \\
\text { (2017) }\end{array}$ & $\begin{array}{l}\text { Estudar a percepção dos cuidadores e } \\
\text { profissionais da psicologia, quanto ao } \\
\text { uso e eficácia das terapias baseado no } \\
\text { método ABA análise } \\
\text { comportamental aplicada) para o } \\
\text { tratamento de pessoas com autismo. }\end{array}$ & $\begin{array}{l}60 \% \text { dos pais e terapeutas consideram que } \\
\text { o nível de apoio necessário as rotinas de } \\
\text { cuidados pessoais diminuem com o tempo } \\
\text { graças à eficácia da terapia. }\end{array}$ & $\begin{array}{l}\text { As estratégias utilizadas pelos } \\
\text { profissionais especialistas geram } \\
\text { mudanças significativas nas áreas } \\
\text { envolvidas em cada caso em } \\
\text { particular, geram melhorias de } \\
\text { competências a esta população } \\
\text { permitindo desenvolver-se em } \\
\text { diferentes contextos e incluir o núcleo } \\
\text { familiar, melhorando sua qualidade de } \\
\text { vida. }\end{array}$ \\
\hline $\begin{array}{l}\text { Souza e Juliani } \\
\text { (2018) }\end{array}$ & $\begin{array}{l}\text { Apresentar as características médicas } \\
\text { do Transtorno e o modelo proposto } \\
\text { pela Análise do Comportamento para } \\
\text { a intervenção. }\end{array}$ & $\begin{array}{l}\text { A Análise do Comportamento Aplicada } \\
\text { temcontribuído enquanto base teórica e } \\
\text { metodológica. }\end{array}$ & $\begin{array}{l}\text { As características médicas do } \\
\text { Autismo e a complexidade e } \\
\text { singularidade de cada } \\
\text { caso exigem um trabalho extenso e } \\
\text { metodologicamente rigoroso, levando } \\
\text { a um constante } \\
\text { repensar das práticas da Psicologia } \\
\text { para esse transtorno }\end{array}$ \\
\hline Matos (2018) & $\begin{array}{l}\text { Mostrar que um tratamento eficaz } \\
\text { para o autismo evidenciando a } \\
\text { Análise do comportamento aplicada }\end{array}$ & $\begin{array}{l}\text { Foi mostrado a abordagem de Skinner que } \\
\text { sinaliza que os comportamentos são } \\
\text { aprendidos e mantidos pelas contingências } \\
\text { de reforço em um determinado ambiente. }\end{array}$ & $\begin{array}{l}\text { A ABA está sendo apontada como um } \\
\text { recurso bastante eficaz para trabalhar } \\
\text { as dificuldades desencadeadas pelo } \\
\text { autismo. }\end{array}$ \\
\hline $\begin{array}{l}\text { Soares e Ferreira } \\
(2017)\end{array}$ & $\begin{array}{l}\text { Analisar o funcionamento autista sob } \\
\text { a ótica da Clínica Infantil Gestáltica. }\end{array}$ & $\begin{array}{l}\text { É possível desenvolver um } \\
\text { acompanhamento marcado por uma } \\
\text { atitude dialógica, que envolve o } \\
\text { acolhimento, presença genuína, aceitação } \\
\text { e respeito do psicoterapeuta para com a } \\
\text { criança, juntamente ao uso de } \\
\text { intervenções, recursos lúdicos, } \\
\text { experimentos e técnicas que se façam } \\
\text { necessários para o desenvolvimento e } \\
\text { crescimento da mesma. }\end{array}$ & $\begin{array}{l}\text { O acompanhamento oferecido na } \\
\text { práxis clínica está ancorado em um } \\
\text { suporte teórico que possibilita uma } \\
\text { relação de acolhimento, aceitação e } \\
\text { respeito ao ritmo e limite da criança } \\
\text { com funcionamento autista, propondo } \\
\text { intervenções que incentivem, na } \\
\text { medida do possível, o seu processo de } \\
\text { contatar de forma funcional. }\end{array}$ \\
\hline $\begin{array}{l}\text { Lopes Neto e } \\
\text { Murador (2020) }\end{array}$ & $\begin{array}{l}\text { Apresentar a Abordagem Centrada na } \\
\text { Pessoa no processo terapêutico de } \\
\text { crianças autistas; enfatizando as } \\
\text { potencialidades do desenvolvimento } \\
\text { apresentado }\end{array}$ & $\begin{array}{l}\text { A terapia centrada no cliente procura, } \\
\text { mais do que explorar e interpretar, } \\
\text { conhecer o mundo interno do cliente. As } \\
\text { funções do terapeuta são de atitude, esta } \\
\text { constitui um patrimônio que vai } \\
\text { compondo a personalidade do terapeuta e } \\
\text { que o leva a tendencialmente perceber e } \\
\text { reagir num determinado sentido }\end{array}$ & $\begin{array}{l}\text { A ludoterapia centrada na criança é } \\
\text { uma modalidade de psicoterapia } \\
\text { infantil eficaz no atendimento da } \\
\text { criança com TEA, pois esta } \\
\text { perspectiva propicia à criança o } \\
\text { contato com quem ela é, com o outro } \\
\text { e com o mundo ao seu redor, a partir } \\
\text { de um encontro permeado pelo } \\
\text { respeito e permissividade para seu } \\
\text { desenvolvimento autêntico. }\end{array}$ \\
\hline
\end{tabular}




\begin{tabular}{|c|c|c|c|}
\hline Brito, et al., (2021) & $\begin{array}{l}\text { Identificar de que forma a terapia } \\
\text { cognitivo-comportamental } \\
\text { auxilia no tratamento e prognóstico } \\
\text { do Transtorno do Espectro Autista } \\
\text { (TEA) }\end{array}$ & $\begin{array}{l}\text { O TEA é estritamente especializado e } \\
\text { direcionado para as principais áreas } \\
\text { afetadas, pois há grande diversidade de } \\
\text { manifestações clínicas para cada paciente. } \\
\text { Atualmente prima-se por intervenções } \\
\text { comportamentais precoces, de forma } \\
\text { intensiva, sendo considerada a terapia } \\
\text { padrão ouro, mundialmente, para o } \\
\text { autismo }\end{array}$ & $\begin{array}{l}\text { Trata-se de um transtorno } \\
\text { comportamental complexo, do } \\
\text { desenvolvimento neurológico, e deve } \\
\text { estar presente desde o nascimento ou } \\
\text { começo da infância, mas pode não ser } \\
\text { detectado antes, devido às demandas } \\
\text { sociais mínimas na mais tenra } \\
\text { infância, e do intenso apoio dos pais } \\
\text { ou cuidadores nos primeiros anos de } \\
\text { vida }\end{array}$ \\
\hline
\end{tabular}

Fonte: Dados da pesquisa (2021).

Considerando as particularidades do Autismo e suas significativas manifestações é relevante o uso de terapias/e ou intervenções que auxiliem no desenvolvimento do indivíduo com TEA, há um leque de sintomas que necessitam de profissionais qualificados e de suas abordagens terapêuticas.

As autoras Steensel e Bögels (2015) falam que os sintomas de ansiedade mais elevados no TEA estão associados a mais problemas comportamentais e mais perturbações da vida, além de dificuldades, proporcionando impacto negativo na qualidade de vida do autista. Relacionado ao tratamento de ansiedade, o comportamento cognitivo (TCC) é considerado eficaz no tratamento de crianças com transtornos de ansiedade e em TEA de alto funcionamento. Alguns estudos também mostraram que o papel da TCC é expandido para abordar outras questões além do TCC como a independência das crianças e habilidades para a vida diária.

As autoras supracitadas asseguram a eficácia da TCC padrão no tratamento do Transtorno de ansiedade em crianças com autismo, ressaltando também a importância de usar o TCC adaptado considerando a habilidade do autista e a questão da medicação que pode interferir parcialmente nos resultados do tratamento utilizado (Ibidem, 2015).

Lopes Neto e Murador (2020) em seu estuda trata sobre a gestalt terapia eles declaram que ela pode enrijecer os limites de contato para crianças com autismo. Porque os Tea possuem dificuldade em estabelecerem relacionamentos satisfatórios entre si e com os outros. Sendo relevante salientar que ambiente externo, grau de dificuldade depende de cada situação e pode afetar o desenvolvimento geral.

A abordagem centrada na pessoa conforme Lopes Neto e Murador (2020) é um desafio, geralmente a família busca resultados a curto prazo, porém o tempo do desenvolvimento e resultado deve ser de acordo com o da resposta da criança, relevante um olhar mais cuidadoso e atencioso, neste aspecto. Considera-se também observar a criança e expandir o seu comportamento pró-social, sabendo que sua comunicação não se limita à expressão verbas, ou seja, sentindo-se mais confiante, seu interesse se amplia, o que confirma seu potencial e a sua existência e só pode ser expressa por condições convenientes.

Contribuindo com a fala dos autores supracitados, Souza e Juliani (2018) destacam também que a psicoterapia para pacientes autistas sempre foi um desafio com base em vários métodos psicológicos, existem várias sugestões de intervenção, a análise comportamental, de enfoque e desenvolvimento aplicações sistemáticas, baseadas no pensamento filosófico que contribuíram para o tratamento do autismo. A combinação de diagnóstico médico e intervenção de análise do comportamento é uma ferramenta importante para lidar com os desafios enfrentados, mesmo sendo um desafio.

Informação importante e que 'grande parte dos estudos defendem é um tratamento do Tea de forma interdisciplinar, considerando a fala de Lopes e Murador (2020), Alvarenga (2017) em sua pesquisa pontua tal observação que as ações devem ser de acordo com o perfil e as habilidades dos indivíduos com autismo. A autora em sua pesquisa ainda relata que entre as 
intervenções mais utilizadas a psicoeducacionais visam o desenvolvimento das habilidades do TEA que estão atrasadas quando comparada com indivíduos neuro típicos.

Conforme Tovar e Rodrigues (2017) a metodologia ABA contribui para o desenvolvimento do TEA, foca em estimular o meio ambiente e o comportamento das crianças para aumentar ou educar diferentes campos, tais como: acadêmico, social, assistência pessoal e redução de comportamento indesejados, são alcançados através das tarefas específicas relacionadas às suas habilidades de comunicação, o foco neste plano alargado e altamente estruturado é baseado em treinamento por meio de tarefas discriminatórias para reduzir o comportamento estereotipado e que incentiva o comportamento socialmente aprovado.

Complementando o posicionamento de Tovar e Rodrigues, De Souza e Juliani (2018), afirmam que a intervenção pelo ABA não requer a realização de um conjunto de tarefas, procedimentos, regras ou etapas fixas e predefinidas, serão intervenções com base nas sete características como: aplicada, comportamental, analítica, tecnológica, conceitualmente sistemática, efetiva, generalizável, as intervenções sempre levam em consideração a personalidade do indivíduo a sua origem social, repertório atual e comportamentos-alvo, enquanto intervenções sistemáticas baseadas na análise de comportamento aplicada geralmente consideram os seguintes quatro aspectos, avaliação comportamental; seleção de alvos; desenvolvimento do plano e intervenção em si / avaliação contínua.

Neste sentido, para Tovar e Rodrigues (2017) a principal função da Análise do Comportamento Aplicada (ABA) é ensinar habilidades importantes de crianças com autismo nas seguintes áreas: atenção, aceitar e expressar linguagem, associação, habilidades motoras boas e abrangentes, Jogos e entretenimento, socialização, autonomia, integração comunitária, Escola e conhecimento escolar.

Ainda conforme as autoras supracitadas em sua pesquisa o propósito principal é de compreender, pensamentos e imaginações sobre a terapia por trás da criação do plano clínico diagnóstico, tratamento e abordagem de atenção integral para indivíduos com Transtorno do espectro do autismo.

Para Matos (2018) o ABA segue os princípios da psicologia comportamental de Skinner, segundo a qual, da perspectiva da regulação do operador, o comportamento é aprendido no processo de interação entre o indivíduo e seu corpo e ambiente social. Frente ao estímulo, em um determinado ambiente, o organismo se comportará e se fortalecerá positivamente, portanto aumentará a possibilidade do mesmo comportamento no mesmo ambiente. A teoria de Skinner propõe que a existência de reforço positivo pode melhorar o comportamento contínuo, enquanto o reforço negativo pode aumentar a resposta para eliminar o comportamento anterior.

Sobre a análise do comportamento autista Matos (2018) diz que requer medidas de intervenção, principalmente para o grupo com os sintomas característicos da síndrome: comunicação social e comportamentos restritos e repetitivos, além de outros comportamentos como acessos de raiva, agressividade e automutilação. Pessoas com autismo podem mudar seus problemas de comportamento e maximizar suas habilidades, de forma que pessoas com autismo possam se desenvolver adequadamente para viver em diferentes ambientes em suas comunidades.

Locatelli e Santos (2016) em seu estudo apresentam o desenvolvimento social com aspecto importante, haja vista que a crianças com autismo são influenciadas por outras, quanto interagem mais aprenderão, ou seja o desenvolvimento está relacionado as interações sociais as pessoas que o autismo têm, tanto o desenvolvimento social, emocional e cognitivo até o seu desenvolvimento sensório-motor., por isso é relevante um ambiente social que deva ser estabelecido conforme os interesses e motivações destes levando em consideração as necessidades, a forma de fornecer informações, interesses e estilo de aprendizagem.

Fazendo uma relação com a pesquisa de Barreto, Nascimento e Andrade (2015) que realizaram sua pesquisa do ponto de vista da psicanálise, enfatizando a dimensão da subjetividade, eles afirmam que se pode considerar que quando a ausência 
do filho e da mãe se alternam, estabelece-se uma verdadeira relação objetal, que é a base para o auto valor. Neste aspecto a importância da intervenção psicoterapia para contribuir no desenvolvimento do autista.

Ou seja, conheça e não me conheça. No caso do autismo infantil, isso é entendido como a falha do tratamento subjetivo e a consciência de si e não-eu sou prejudicada, sendo possível apontar a evolução da situação clínica dessas crianças ao longo de um tratamento, processo pode se revelar em uma maior abertura para os outros e no uso simbólico e funcional desses objetos (Ibidem, 2015).

Ainda, conforme os autores (Ibidem, 2015) no campo da psicanálise, é muito importante realizar pesquisas voltadas para a reflexão sobre os objetos utilizados pelas crianças autistas, pois esses dados podem trazer elementos básicos para o seu processo subjetivo. Os objetos autistas têm funções infantis em vez de enfrentar um vácuo. A prática é suprema, podendo reiterar e / ou questionar as teorias existentes. O desafio para esta clínica de autismo é manter a disposição para interagir com outras pessoas sob as mais ricas nuances do ser humano e respeitar o tempo do paciente e, claro, usar a criatividade para lidar com inúmeras frustrações e potenciais.

Na terapia cognitivo-comportamental, (Alvarenga, 2017) o tratamento é baseado na reconstrução cognitiva, por meio da mudança de crenças, pensamentos disfuncionais e comportamentos subsequentes. Nos modelos cognitivos, as pessoas pensam que o que move nossas vidas são os nossos pensamentos, ou seja, nós como explicar o que aconteceu. Portanto, o pensamento produz emoção, que produz comportamento. É importante enfatizar que os modelos cognitivos não podem ser vistos de forma linear como situações que desencadeiam pensamentos, que podem desencadear emoções e, portanto, comportamentos.

Alvarenga (2017) ainda pontua às habilidades sociais, afirma que elas devem ser ensinadas em um ambiente que seja significativo para os sujeitos, e sua motivação para o treinamento deve ser fortalecida por meio de técnicas de TCC (como experimentos comportamentais, reforço ativo e reconstrução cognitiva).

Além disso, o programa de treinamento de habilidades sociais deve ser específico para cada situação e levar em consideração os pontos a serem desenvolvidos e priorizados, como compreensão social, regras de interação social e aprendizagem de expectativas interpessoais, diálogo e equivalência, uso não verbal e interpretação de personagem Autocontrole e comportamento destrutivo, comportamento repetitivo e estereotipado e transtorno obsessivo-compulsivo formam amizades.

Brito et al., (2020) A terapia cognitivo-comportamental (TCC) é uma forma de tratamento mais completa porque melhora o escopo da psicopatologia nos aspectos cognitivos e comportamentais. A TCC é um conjunto de atividades que auxiliam no tratamento da psicopatologia, portanto, seus métodos e objetivos conceituais provêm principalmente de dois métodos: o comportamental e o cognitivo, que serão avaliados a partir do exercício integral da psicologia, que dá origem à chamada cognição Terapia-Comportamento Terapia.

A pesquisa de Soares e Ferreira (2017) tratam da Gestalt-terapia que sobre o autismo possui algumas considerações como: a perturbação da função id pode fazer com que o limite das seguintes situações endureça; Interrupções durante o ajuste e contato estábulo e acompanhamento fornecido. Na prática clínica, baseia-se em suporte teórico e pode fazer Aceite, aceite e respeite a relação entre o ritmo e os limites da criança $\mathrm{O}$ funcionamento de pacientes autistas e propor intervenções para encorajá-los tanto quanto possível.

Os autores supracitados ainda completam que a Gestalt-terapia concebe o processo de contato de uma forma funcional A função de contato está incorporada na fronteira de contato, ou seja, o local de troca, o indivíduo e o meio ambiente fluem na assimilação de coisas novas, nutritivas ou novas. Recuse-se a usar coisas prejudiciais como o ponto central de compreensão este tópico. Com essa abordagem, o contato é essencial para o crescimento. Por meio dela, os seres humanos podem se estabelecer. 
Assim, Soares e Ferreira (2017) afirmam que é importante notar que a Gestalt terapia não tem nada a ver com o "porquê" (ou seja, a causa do autismo ou doença), mas com o "como" (o tratamento nele). O paciente autista pode enfrentar suas próprias limitações e dificuldades por meio de possíveis ajustes criativos, não apenas do ponto de vista da psicopatologia, mas também para compreender sua situação geral em seu próprio campo e interação.

Com relação aos aspectos necessários nesta abordagem a pesquisa realizada pelos autores destacou a necessidade de abrir mão do próprio comportamento autista, para compreender melhor a condição da criança, a fim de se ter uma compreensão mais abrangente dela (Ibidem, 2017).

Para os pesquisadores supracitados no processo de psicoterapia são utilizados métodos fenomenológicos, que se caracterizam pela linguagem descritiva, e a intervenção do terapeuta não requer a priori. Esta também utiliza recursos lúdicos, tecnologias e experiências para promover a sua expressão e comunicação no espaço de tratamento. Os recursos técnicos utilizados na psicoterapia infantil são diversos e têm como principal objetivo trabalhar e explorar as funções infantis. Portanto, por meio da tecnologia da psicoterapia infantil, também é possível ajudar amplamente as crianças com distúrbios do ciclo de contato a se perceberem melhor com seus próprios sentimentos e sentimentos quando estão em contato consigo mesmas.

\section{Conclusão}

A partir dos resultados obtidos nesta pesquisa, considera-se alcançados os objetivos do estudo, haja vista que os artigos selecionados, apresentam em seu corpo informações relevantes sobre estratégias, metodologias, abordagens eficazes e diante destas intervenções certas mudanças de comportamento e os sintomas do autismo melhorando sua qualidade de vida.

Nos métodos cognitivo-comportamentais, acredita-se que crianças com autismo tenham as mesmas habilidades que crianças com neuróticas em intensidades diferentes. A experiência em psicanálise afirma que o espaço de tratamento pode proporcionar a essas crianças autistas novas oportunidades, para que possam avançar subjetivamente no momento em que sua estrutura psicológica ainda não está fechada e decidida.

O objetivo da terapia cognitivo-comportamental no tratamento do autismo é adaptar os sujeitos por meio do desenvolvimento de habilidades, na intervenção o modelo cognitivo-comportamental busca realizar um trabalho do terapêutico como base no entendimento do comportamento dos indivíduos para alcançar um desempenhos bem-sucedidos de suas habilidades.

A Gestalt-terapia não ignora esse conceito, mas parte de um pressuposto que traz uma perspectiva mais abrangente, por não se basear em fatores específicos, e tenta compreender o paciente de forma abrangente, em vez de fazê-lo adoecer. Esse processo pode ser viabilizado por meio da Gestáltica para buscar ampliar as fronteiras de contato e os recursos internos da criança, reduzir a rigidez e caminhar em direção a uma maior mobilidade, além de despertar a consciência e se encorajar a se ver como uma pessoa diferente e única em um mundo que está em constante mudança e em contato constante com os outros, as necessidades são atendidas.

Explorar suas funções de contato e manter contato consigo mesmo, buscar o diálogo com eles, e estimulá-los a brincar e se descobrir a todo momento, para que o caminho antes estreito e de pedra comece a se alargar e se desenvolver na direção do contato e do crescimento. O mais rápido possível esse processo pode ser realizado por meio da Clínica Infantil Gestáltica para buscar ampliar o alcance e os recursos internos da criança para diminuir a rigidez e caminhar em direção a uma maior mobilidade, despertar a consciência e estimular a autopercepção. Neste mundo em constante mudança e contato constante com os outros, as necessidades e necessidades de uma pessoa única são atendidas.

No decorrer do estudo foram citadas várias técnicas interventivas, sobretudo após a adoção da Terapia Cognitivo Comportamental (TCC) que traz em suas práticas uma certa tranquilidade para os envolvidos no tratamento. Os resultados encontrados nos mostram a eficácia do tratamento ABA como grande benefício para as crianças com TEA. 
As melhorias nos métodos para pacientes autistas envolvem principalmente habilidades adaptativas, linguagem, habilidades sociais e funções executivas relacionadas ao contato visual, seguir instruções, permanecer à mesa, regulação do humor, rotinas de cuidados pessoais, análise comportamental aplicada e autismo.

Portanto, considera-se alcançado o objetivo da pesquisa, haja vista que os artigos e teses apresentadas contribuem para mostrar pontos relevantes sobre as abordagens terapêuticas psicologia para o TEA autismo.

\section{Referências}

Alvarenga, G. C. S. (2017). Autismo leve e intervenção na abordagem cognitivo-comportamental. Especialização em Terapia Cognitivo- comportamental. Centro de Estudos em Terapia Cognitivo-Comportamental - CETCC. São Paulo.

Assunção, W. C.; Silva, J. B. F. (2019). Aplicabilidade das técnicas da terapia cognitivo-comportamental no tratamento de depressão e ansiedade. Revista Educação Psicologia e Interface, Cuiabá, v. 3, n.11, p. 77-99, jan./ago.

Barreto, D. V. C. A.; Nascimento, K.N.; Andrade, F. W. C. A. (2015). Como podemos situara os objetivos autísticos e transicionais na clínica psicanalíticas do autismo? Revista FAIFIRE, Recife, v, 8 n.2, p. 56-77, jun./dez.

Bezerra, M. F. (2018). A importância do método aba - reanálise do comportamento aplicada - no processo de aprendizagem de autistas. Revista Científica Multidisciplinar Núcleo do Conhecimento, São Paulo, v. 06, p. 189- 204, out.

Brito, M. et al. (2021). O impacto da terapia cognitivo-comportamental no transtorno do espectro autista. Brazilian Journal of Health Review, Curitiba, v.4, n.2, p. 7902-7910 mar. /apr.

Chaim, M. P. M. et al. (2020). Fenomenologia da qualidade de vida de mães de crianças autistas. Revista Abordagem Gestáltica, Goiânia, v. 26, n. 2, p. 122134, ago. http://pepsic.bvsalud.org/scielo.php.

Goncalves, A. P. et al. (2017). Transtornos do espectro do autismo e psicanálise: revisitando a literatura. Tempo psicanálise, Rio de Janeiro, v. 49, n. 2, p. 152181, dez. http//pepsic.bvsalud.org/scielo.php.

InstitutoNeurosaber. (2017). Como é a Intervenção com autismo na Análise Aplicada do Comportamento (ABA). Outubro. https://institutoneurosaber.com.br/artigos/neurosaber/.

Jerusalinsky, A. (2017). Considerações preliminares a todo tratamento possível do autismo. Psicologia Argumento, [S.1.], v. 28, n. 61, nov. https://periodicos.pucpr.br/index.php/psicologiaargumento/article/view/19695/19023.

Lopes, N. M. A; Murador, F. S. (2020). Ludoterapia e a criança autista versus a abordagem centrada na pessoa. Revista Acadêmica-online. http://files.revistaacademica-online.webnode.com/200000678-801c8801cb/artcient22082020.pdf.

Matos, R. S. P. (2018). As Dificuldades de Aprendizagem em Pessoa com Autismo e as Contribuições da Análise do Comportamento Aplicada-ABA. JournalofSpecialist, [S.1.], v. 1v.4, n.4, p.3-20, Out-dez.

Paiva Júnior, F. (2021). O que é autismo. Revista Autismo, São Paulo, ano VI, n.11, p.8, fev.

Soares, M. N.; Ferreira, B.N.W. (2018). O funcionamento autista sob a ótica da clínica gestáltica. Revista do NUFEN. [S.I.] v.10, n.2, p. 75-90, ago.

Souza, R. D. B.; Juliani, J. (2018). Psicologia e autismo. Revista Terra \& Cultura: Cadernos de Ensino e Pesquisa, [S.1.], v. 29, n. 56, p. 139-152, jul. 2018. ISSN 2596-2809.http://periodicos.unifil.br/index.php/Revistateste/article/view/194.

Steensel, F. V. Bögels, S. M. (2015). TCC para transtornos de ansiedade em crianças com e sem autismo Distúrbios do espectro. Jornal de Consultoria e Psicologia Clínica. Psicologia Clínica, [S.I] v. 83, n. 3, p. 512-523. fev.

Teixeira, G. (2016). Manual do Autismo. Rio de Janeiro: Best Seller.

Tovor, L. M. C; Rodriguez, S. A.L. (2017). Percepción de cuidadores y profesionalesenpsicología, frente al uso y efectividad de terapias basadasenel método aba (appliedbehavioranalysis) para eltratamiento de personas con autismo. Iberoamericanacorporacion universitária. 